\title{
Assessment of bone mineral density in multi-transfused thalassemia patients in a tertiary care hospital
}

\author{
Shah S. ${ }^{1}$, Shah J. ${ }^{2 *}$, R. Vyas B. ${ }^{3}$ \\ DOI: https://doi.org/10.17511/ijpr.2020.i01.06
}

1 Sonal Shah, Additional Professor, Department of Pediatrics, G.G.G. Hospital, Jamnagar, Gujarat, India.

2* Jil N. Shah, Senior Registrar, Department of Pediatrics, Gmers Medical College and Civil Hospital, Ahmedabad, Gujrat, India.

3 Bhadresh R. Vyas, Professor, Department of Pediatrics, G.G.G. Hospital, Jamnagar, Gujrat, India.

Objective: Patients with transfusion-dependent thalassemia (TDT) are susceptible to osteopenia and osteoporosis, the mechanism is multi-factorial. Transfusion-related iron overload in endocrine organs leads to impaired hormonal secretion, all contributing to bone loss. The aim is the assessment of bone loss frequency and related parameters affecting it in TDT patients in tertiary care Centre, Jamnagar. Design: Observational cross-sectional study. Subjects: 33. Methods: The study is conducted over 1 year in TDT patients attending G.G.G. hospital for blood transfusion. After clearance from the ethical committee and consent from parents, patients selected according to inclusion criteria and scanned for Bone Mineral Density (BMD) and biochemical tests. Results analyzed with the help of standard statistical tests. Results: Out of 33 patients, 36\% (12) patients had osteopenia while 45\% (15) patients had osteoporosis. $84 \%$ (16) of 19 male patients and $78 \%$ (11) of 14 female patients had low BMD. 80\% (4) of 5 patients with high S.TSH levels and all patients with low calcium levels, were detected with low BMD.11\% (3) of 27 patients having low BMD, had high S. Alkaline phosphatase level. 85\% (6) of 7 patients with low S.FSH levels and $73 \%$ (8) of 11 patients with low S.L.H. levels have low BMD. 87\% (14) of 16 patients with short stature have low bone mass. Conclusion: Evaluating BMD annually in TDT patients helps prevent and intervene timely. Administration of bisphosphonates, calcium, vitamin-D supplements, hormone replacement therapy, bone-forming agents and newer therapies will help improve bone mass. Changing lifestyle with appropriate diet and exercise, iron chelation therapy, early diagnosis and treatment of endocrine insufficiency and regular blood transfusions help achieve optimal bone density.

Keywords: Bone loss, Bone mineral density, Osteoporosis, Thalassemia

Corresponding Author

Jil N. Shah, Senior Registrar, Department of Pediatrics, Gmers Medical College and Civil Hospital, Ahmedabad, Gujrat, India.

Email: jilsheth89@gmail.com
How to Cite this Article

To Browse

Shah S, Shah JN, Vyas BR. Assessment of bone mineral density in multi-transfused thalassemia patients in a tertiary care hospital. Pediatric Rev Int J Pediatr Res. 2020;7(1):32-38.

Available From

https://pediatrics.medresearch.in/index.php/ijpr/arti cle/view/559

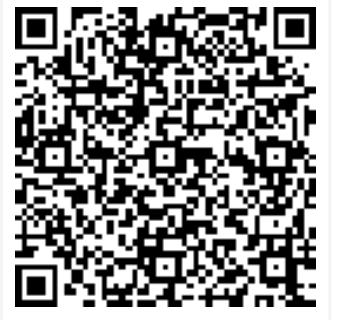

Manuscript Received 2020-01-10

Conflict of Interest No
Review Round 1 2020-01-20

Funding Nil

Review Round 2
$2020-01-25$
Ethical Approval
Yes

Review Round 2

Yes
Review Round 3

Plagiarism X-checker $9 \%$
Accepted 2020-01-28

Note

(c) 2020by Sonal Shah, Jil N. Shah, Bhadresh R. Vyas and Published by Siddharth Health Research and Social Welfare Society This is an Open Access article licensed under a Creative Commons Attribution 4.0 International License https://creativecommons.org/licenses/by/4.0/ unported [CC BY 4.0]. 


\section{Introduction}

Thalassemia is one of the most common single-gene disorders; it is inherited as an autosomal recessive disorder. Every year approximately 1 lakh children with thalassemia major are born world over, of which 10,000 are born in India [1]. Certain communities in India such as Kutchis, Sindhis, Punjabis, Bhanushalis, Lohanas, Mahars, Neobuddhists, Gowdas, etc. have a higher carrier state. In Gujarat, the prevalence is $1.95 \%$ i.e. 10,000 children approximately, with higher frequency noted in Bhanushalis and Bhakta communities in Banaskatha district [2]. Every year 1000 new patients enter the world of thalassemia in Gujarat.

The thalassemia syndromes are a heterogeneous group of inherited anaemias characterized by defects in the synthesis of one or more globin chain subunits of the adult haemoglobin tetramer $(\mathrm{HbA})$, this leads to deficient haemoglobin resulting in hypochromic microcytic red cells, ineffective erythropoiesis and hemolytic anaemia. Blood transfusions therapy on a continuing basis represents the primary treatment for $\beta$ thalassemia. Repeated blood transfusion leads to massive tissue deposition of iron and subsequently on long term basis causes multiorgan dysfunction as a complication of it. In spite of the improved treatment of the hematologic disorder and its complications, $\beta$ thalassemia patients exhibit an imbalance in bone mineral turnover with increased resorptive rates and suppression of osteoblast activity, resulting in diminished bone mineral density (BMD) more evident in the lumbar spine [3].

Osteoporosis is a disease characterized by low bone mass and micro-architectural deterioration of bone tissue, leading to enhanced bone fragility and a consequential increase in fracture risk [4]. Untreated transfusion-dependent thalassemia (TDT) patients present with severe bone deformities, associated with osseous changes, in particular, facial and limb deformities that have been attributed to bone marrow expansion and cortical thinning due to ineffective erythropoiesis [5]. Incidence of osteoporosis in TDT ranges from $44-96 \%$ and fracture rates are $70 \%$ higher in thalassemia when compared to the general population. TDT suffer from bone pain and low bone mass [6]. Osteoporosis thus, represents a prominent cause of morbidity in TDT in both genders [7].
Several acquired and genetic factors play a role. Chronic hypoxemia and medullary expansion due to low baseline haemoglobin, defective growth and nutritional status, abnormal calcium-phosphate homeostasis, increased levels of iron, delayed puberty and hypogonadism are of particular importance. Clinical features include growth failure, osteoporosis, fractures, osteonecrosis of bones, bone and spinal deformities, nerve compression. A common symptom is back pain which could be due to osteoporosis, fractures, disc disease due to long term desferrioxamine toxicity and extramedullary hematopoietic masses. Diagnosis is done by DEXA scan (dual Xray absorptiometry) of the lumbar spine and femoral neck [8]. The DEXA technique analyses the attenuation of Xrays as they pass through an area of the body. The cut-off of 2.5 standard deviations below the normal mean in BMD for the respective age is used for the definition of osteoporosis, whereas a decrease of BMD between 1 and 2.5 standard deviations below the normal mean for the respective age is defined as osteopenia [9]. Other methods are quantitative computed tomography (QCT), Magnetic resonance imaging (MRI) of the spine, various biochemical tests such as S. Calcium, S. phosphorus, Alkaline phosphatase and vitamin $D$ levels.

Prevention and general measures include annual checking of BMD starting in adolescence with regular physical activity, adequate hormonal replacement, effective iron chelation, improvement and maintenance of hemoglobin levels, calcium and vitamin D supplementation [10]. Bisphosphonates are the first choice of therapy in children with osteoporosis without hypogonadism and second choice in children with hypogonadism on hormone replacement therapy (HRT). They decrease bone resorption by inhibiting osteoclastic recruitment and maturation, preventing the development of monocyte precursors into osteoclasts, inducing osteoclast apoptosis and interrupting their attachment to bone. The common side effects are vomiting, giddiness, nephrocalcinosis, and sometimes on long term therapy, osteonecrosis of the jaw. Alendronate, Pamidronate, Zoledronic acid, the most potent third-generation bisphosphonate are some of the examples. Calcitonin is a potent osteoclast inhibitor, has anabolic effects on cartilage formation, bone matrix synthetic activity and bone growth. Bone forming agents like teriparatide and strontium ranelate and recombinant PTH and newer agents like hydroxyurea and antibodies against 
RANKL, such as denosumab, and antibodies against Dkk-1 or sclerostin are under study.

The rationale of the study is early detection of low BMD by evaluating it annually and regularly investigating through various biochemical tests. This will allow timely and appropriate bone resuscitative measures to be undertaken and finally, improvement in the quality of life of these patients with minimal intervention. The outcome of the results will also help us know the effect of various factors affecting the bone mineral density, correlating it with the symptomatology of the patients and will help patients for starting the treatment and the policymakers for designing policies regarding therapeutic and preventive aspects.

The aims and objectives are to study the assessment of bone mineral density in multitransfused thalassemia patients through DEXA scan and to assess the factors affecting bone mineral density in them.

\section{Methods}

Setting: Guru Gobind Singh Government Hospital, Jamnagar, Gujarat, India

Duration: 1 year from July 2014 to June 2015

Type of study: Observational cross-sectional study

Sampling Method: Not applicable

Sample size calculation: not applicable

\section{Sample size: 33}

Inclusion criteria: All thalassemia patients who had $\geq 50$ transfusions till date, with/without symptoms related to low bone mass, who are not receiving any supplementations affecting bone mineral density, who consent to the study and with age group from 8-18 years are included in the study.

Exclusion criteria: Transfusion dependent thalassemia patients who had $<50$ transfusions till date, who don't consent to the study and patients with age group $<8$ years and $>18$ years.

Data collection procedure: Data of selected patients was collected and recorded in a proforma with emphasizing on the basic information of the patients, biochemical reports like S. Ferritin, S. Calcium, S. alkaline phosphatase, S. TSH, S. FSH,
S. LH, S, testosterone, S. estradiol and general physical condition of the patient. The patients were then sent for DEXA scan to the Radiology Department, G.G.G. Hospital, Jamnagar. After consenting for the scan, patients were asked to wear loose comfortable clothes, avoid garments with zippers, belt or buttons.

Jewellery, keys, wallets, or any other metal object that would interfere with the test was advised to be removed. Scan was performed with central DEXA device of General Electric company (Lunar DPX DXA System: analysis version-12.30). This examination was done on an outpatient basis. The DEXA bone density test usually takes 10 to 30 minutes.

Data analysis: The entire data was collected for every patient as per the proforma and analysis of the effect of various parameters on bone mineral density was done like iron overload, hypothyroidism, hypoparathyroidism, lack of sex steroids, vitamin D deficiency, etc. For Qualitative data, Pearson Chisquare test and Fisher exact test were used with continuity correction wherever applicable with the help of Epi Info software, version 3.5.3.

\section{Ethical consideration and permission:} Institutional Ethical committee for research on human subjects gave approval and clearance for the study protocol. Written informed consent of parents or guardian and assent (for those $>12$ years) was obtained for participation in the study prior to evaluation of their child.

Scoring System: The test results were recorded in the form of $Z$ score - shows the amount of bone to be compared with that of a young adult of the same gender with peak bone mass. WHO defines a score above -1 as normal. A score between -1 and -2.5 is classified as osteopenia (low bone mass). A score below -2.5 is defined as osteoporosis. The $Z$ score is used to estimate the risk of developing a fracture.

\section{Results}

Patients were assessed of their bone mineral density (BMD) with the help of DEXA scan. The results are interpreted in the form of $Z$ score. A score above -1 is considered normal. Low BMD includes both osteopenic and osteoporotic range i.e. $\mathrm{Z}$ score $\leq-1$ [3].

A total of 33 patients were assessed of their bone mineral density with the help of DEXA scan, along with the related parameters affecting it. 
- Out of a total 33 patients, 27 were found to have low BMD i.e. 12 (36\%) patients had osteopenia while $15(45 \%)$ patients had osteoporosis.

- 16 of the $19(84 \%)$ male patients suffered from low bone mass while 11 of the $14(78 \%)$ female patients had the same.

- 4 of the $5(80 \%)$ patients with high S.TSH levels have been found of low bone mineral density.

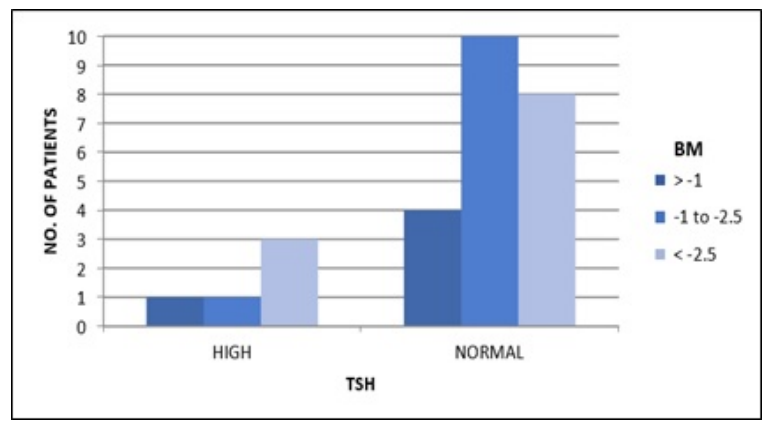

Fig-1: Distribution of TSH levels in patients.

- $5 \%$ patients having $<150$ transfusions, $85 \%$ patients having transfusions between 150-250, $80 \%$ patients having transfusions $>250$ suffer from low BMD.

- All those TDT patients found to have low calcium levels, have been detected with low BMD with $75 \%$ in the osteopenic range.

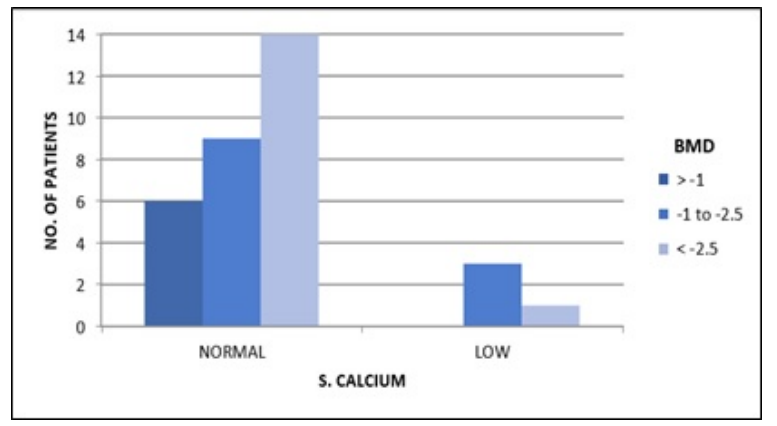

Fig-2: Distribution of Serum Calcium levels in patients.

- 3 of the 27 (11\%) patients having low BMD, are found to have high $\mathrm{S}$. Alkaline phosphatase level.

- 6 of the $7(85 \%)$ patients with low S.FSH level and $100 \%$ of the patients with high S.FSH levels suffer from low BMD.

- 8 of the $11(73 \%)$ patients with low S. LH levels and $100 \%$ of the patients with high S. LH levels suffer from low BMD.
- $100 \%$ of the patients with low S. Testosterone levels were found to have low BMD.

- 14 of the $16(87 \%)$ patients with short stature suffered from low BMD.

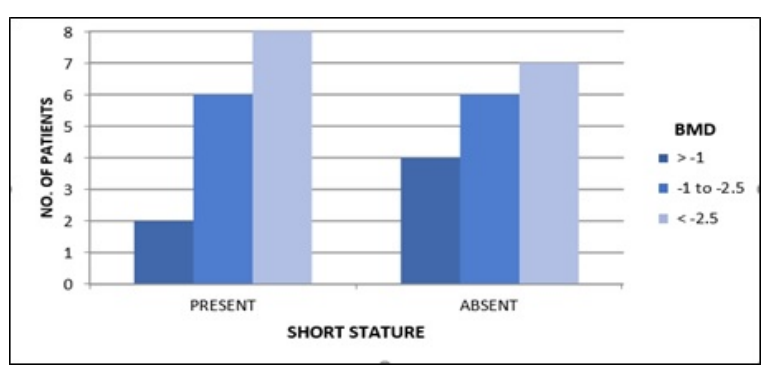

Fig-3: Distribution of patients with short stature.

\section{Discussion}

Thalassemia bone disease (TBD) is a common and severe complication of thalassemia-an inherited blood disorder due to mutations in the a or $\beta$ haemoglobin gene. In its more severe form, severe anaemia is present, and treatment with frequent red blood cell transfusion is necessary. Because the body has limited capacity to excrete iron, concomitant iron chelation is required to prevent the complications of iron overload. The effects of chronic anemia and iron overload can lead to multiple end-organ complications such as cardiomyopathy, increased risks of blood-borne diseases, and liver, pituitary, and bone disease.

However, our understanding of thalassemia bone disease is incomplete and is composed of a complex piecemeal of risk factors that include genetic factors, hormonal deficiency, marrow expansion, skeletal dysmorphism, iron toxicity, chelators, and increased bone turnover. The high prevalence of the bone disease in transfusion-dependent thalassemia is seen in both younger and older patients as life expectancy continues to improve. Indeed, hypogonadism and $\mathrm{GH}$ deficiency contribute to a failure to achieve peak bone mass in this group. Thalassemia bone disease is a composite of not only multiple hormonal deficiencies but also multiorgan diseases [11].

Conventional treatment of thalassemia, namely regular blood transfusion and iron chelation, improves perspectives and quality of life; however, successful treatment leads to more time in which long-term complications such as bone disease can develop. 
Thalassemia bone disease is unique: all aspects, from bone anatomy and bone quality to mineral density, may be affected, with important morbidity including osteoporosis, fractures, spinal deformities, nerve compression, and pain. Clinical presentations include growth impairment, rickets-like features, back pain, spinal deformities, any sign of nerve compression, severe osteoporosis, and fragility fractures. Age, history, physical examination, and diagnostic tests support orientation on risk factors. These include bone marrow expansion, toxicity from iron overload and iron chelation, endocrine dysfunctions (hypogonadism, hypo/ hyperparathyroidism, hypothyroidism, growth hormone deficiency, diabetes), and vitamins (D, C, $\mathrm{K}$ ) and zinc deficiencies.

Several of these may coexist in an individual for a long time and at different degrees, making a clarification of the relative contribution and selection of the best therapeutic options a challenge. Milestones for prevention of TBD are early and full inhibition of bone marrow hyperplasia and iron toxicity. Empowering patients' positive resources is key for achieving long-term healthy habits with regard to diet, physical activity, sunlight exposure, and lifestyle [12].

The majority ( $82 \%$ ) of the TDT patients suffer from low bone mineral density and thus represents a prominent cause of morbidity. Low bone mass is of multifactorial etiology and of complex mechanism which includes compensatory massive expansion of hyperplastic bone marrow, secondary hemochromatosis, endocrine abnormalities, vitamin deficiencies, reduced physical activity and some genetic mutations. It was found that $16(84 \%)$ out of 19 male patients while $11(78 \%)$ out of 14 female patients suffering from low BMD. Abbasi $S$ [13] et al determined bone mineral density (BMD) of patients with beta-thalassemia in order to find the prevalence and related factors on the conditions. ZScore of the femoral neck and lumbar vertebrae were reported. It was found the disease had significantly higher severity in men than in women. There were significant differences between sex hormone and bone density. Osteopenia and osteoporosis were highly prevalent in the participants. Therefore, regular tests are required to examine bone mineral density in these patients.

$M$ Karimi [9] et al. conducted study to assess BMD in 106 thalassemia patients using DEXA scan and correlated with the biochemical and haematological profile.
The results showed lower BMD and BMC in the lumbar region ( $p<0.05)$. Both parameters correlated significantly with haemoglobin level; other biochemical and haematological parameters did not influence BMD and BMC values.

$80 \%$ of patients having high S.TSH levels have low BMD. High S.TSH i.e. hypothyroidism (HT) may occur due to chronic tissue hypoxia or poorly chelated iron overloaded patients. Shamshirsaz AA [14] et al. did a multicenter study on metabolic and endocrine complications in beta-thalassemia major in Tehran in which 220 patients were examined and primary hypothyroidism was found in $7.7 \%$ patients. A Bazi [15] et al. assessed the frequency of HT and hypoparathyroidism (HPT) in a population of thalassemia patients in Southeast of Iran. A total of $103(53.1 \%)$ and 91 (46.9\%) females and males were recruited in this study, respectively. HT and HPT showed overall frequencies of $8.2 \%(18 / 194)$ and $18 \%(35 / 194)$, respectively.

As the number of transfusions increases, it was found to affect BMD. The present can see that $78.5 \%$ patients having $<150$ transfusions, $85 \%$ patients having transfusions between 150-250, 80\% patients having transfusions $>250$ suffer from low $B M D$, With increasing number of transfusion there is deposition of iron in the body with no efficient mechanism for its excretion, thus leading to secondary hemochromatosis within tissues like bone and endocrine glands. This all affects bone metabolism leading to various forms of bone deformities and morbidities. In this study, it was observed that none of the patients suffered from symptoms of osteoporosis which might be due to well chelation of iron and regular transfusions!

It was observed that all those patients found to have low serum calcium levels suffer from low BMD which may be due to secondary hemosiderosis in the parathyroid gland, owing to suppression of parathyroid secretion and thus a low serum calcium level. This might also be due to low levels of vit D3. Also $11 \%$ of patients with low BMD showed high S. Alkaline Phosphatase levels. The low bone mass is due to increased bone resorption, thus shooting the S. Alkaline phosphatase levels high. Fathy Khaleel Nawar [16] studied to delineate calcium status and bone mineral density in a group of transfusiondependent Egyptian $\beta$ thalassemia patients of both sexes. The results obtained showed that $S$. Calcium was lower, Alkaline Phosphatase was higher as compared to the control group. 
$85 \%$ of patients having low S.FSH levels and $100 \%$ of patients having high S.FSH levels suffer from low BMD. Iron deposition in the pituitary gonadotrophic cells leads to disruption of gonadotropin (LH, FSH) production. Occasionally gonadal iron deposition occurs leading to secondary hypogonadism. Thus, gonadal dysfunction in the form of hypogonadism or delayed puberty affects bone maturation and bone mineral content. $73 \%$ of the patients having low S. LH levels while $100 \%$ of patients having high S. LH levels suffer from low BMD. $100 \%$ of patients having low S. Testosterone levels suffer from low BMD. Dundar $U$ [17] studied bone metabolism and mineral density in patients with $\beta$ thalassemia major in Turkey, to evaluate and determine factors associated with the development of osteoporosis. BMD of the lumbar spine and proximal femur were determined in 25 patients along with 24 controls. Serum levels of estradiol and progesterone in females, luteinizing hormone and follicle-stimulating hormone in both genders were significantly lower in patients.

$88 \%$ patients with short stature suffer from low BMD. Secondary hemosiderosis in endocrine organs like pituitary, gonads, thyroid and pancreas, along with poorly managed diet may all lead to growth failure. Or low BMD due to multiple reasons may itself lead to short stature. Ibrahim Aslan [18] studied bone mineral density and related parameters in nine adults, thirty-eight pubertal, prepubertal, with a total of forty-seven patients with thalassemia major from Antalya, Turkey. The study assessed BMD and height. A moderate relationship was found between the bone mass density age and height. Subjects in low pubertal staging and short stature $(<3 \%$ percentile) have significantly lower bone mass densities.

The limitations of the present study are: limited sample size, the ineffectiveness of DEXA scan in predicting the fracture risk as well as for patients with spinal deformity.

\section{Conclusion}

Osteoporosis is a progressive disease and it has a multifactorial pathogenesis in TDT patients. In this study, 33 patients were evaluated from the age group of 8-18 years for bone mineral density. Our results showed that the majority of TDT patients suffer from low bone mass but were symptomless due to better, regular chelation and blood transfusions.
The present study found that many patients with low S.TSH levels, low S. Calcium levels, low Testosterone levels and short stature suffered from low BMD.

Policymakers need to have a larger sample size for significant results to identify parameters affecting low bone mass and thus preventing and appropriately treating them. With recent protocols increasing the life expectancy of TDT, osteoporosis and osteopenia form a major cause of morbidity in these patients and hence warrant early intervention for its prevention and management.

\section{What does this study add to existing knowledge?}

With high prevalence of thalassemia related bone disease and its related morbidity, to prevent it, annual BMD should be done regularly starting from prepubertal period along with appropriate bone resuscitative measures like bisphosphonates, HRT and bone-forming agents, adequate hormonal replacement, effective iron chelation, improvement of $\mathrm{Hb}$ levels, calcium and vitamin D administration, physical activity to improve the quality of life of these patients.

\section{Author's contributions}

Dr. Sonal Shah: Concept, study design. Dr. Jil N. Shah: Data analysis, manuscript preparation. Dr. Bhadresh R. Vyas: Manuscript preparation

\section{Reference}

01. Verma IC. Burden of genetic disorders in india. Indian J Pediatr. 2000;67;893-898. doi: 10.1007/BF 02723953 [Crossref][PubMed][Google Scholar]

02. Patel Ashwin $P$, Naik Madhuben $R$, Shah Nilam M, Sharma Narmadeshwar P, Parmar Prakash H. Prevalence of common hemoglobinopathies in gujarat- an analysis of a large population screening program. Nat J Comm Med. 2012;3(1)112-114. [Crossref][PubMed][Google Scholar]

03. Mahachoklertwattana $P$, Chuansumrit $A$, Sirisriro R, Choubtum L, Sriphrapradang A, Rajatanavin R. Bone mineral density, biochemical and hormonal profiles in suboptimally treated children and adolescents with beta-thalassaemia disease. Clin Endocrinol (Oxf). 2003;58(3)273-279. doi: 10.1046/j.1365-2265.2003.01707.x [Crossref] [PubMed][Google Scholar] 
04. Assessment of fracture risk and its application to screening for postmenopausal osteoporosis. Report of a WHO Study Group. World Health Organ Tech Rep Ser. 1994;843;1-129. Available at: [Article] [Crossref][PubMed][Google Scholar]

05. Cooley TB, Lee P. A series of cases of splenomegaly in children with anemia and peculiar bone changes. Trans Am Pediatric Soc. 1925;37;2930. [Crossref][PubMed][Google Scholar]

06. Dresner Pollack R, Rachmilewitz E, Blumenfeld A, Idelson $M$, Goldfarb AW. Bone mineral metabolism in adults with beta-thalassaemia major and intermedia. $\mathrm{Br}$ J Haematol. 2000;111(3)902907. doi: 10.1111/j.1365-2141.2000.02392.x [Crossref][PubMed][Google Scholar]

07. Voskaridou E, Terpos E. New insights into the pathophysiology and management of osteoporosis in patients with beta thalassaemia. $\mathrm{Br} \mathrm{J}$ Haematol. 2004;127(2)127-139. doi: 10.1111/j.13652141.2004.05143.x [Crossref][PubMed][Google Scholar]

08. Karimi M, Ghiam AF, Hashemi A, Alinejad S, Soweid $M$, Kashef $S$. Bone mineral density in betathalassemia major and intermedia. Indian Pediatr. 2007;44(1)29-32.

[Crossref][PubMed][Google Scholar]

09. Arjmandi Rafsanjani K, Razzaghy-Azar $M$, Zahedi-Shoolami L, Vossough $\mathrm{P}$, Modarres A, Taheri $N$. Bone Mineral Density in $\beta$ Thalassemia Major and Intermedia, Correlation with Biochemical and Hormonal Profiles. Iran J Blood Cancer. 2009;1(4)121-127. doi: [Article][Crossref][PubMed] [Google Scholar]

10. Terpos E, Voskaridou E. Treatment options for thalassemia patients with osteoporosis. Ann N Y Acad Sci. 2010;1202;237-243. doi: 10.1111/j.1749-6632.2010.05542.x [Crossref] [PubMed][Google Scholar]

11. Wong P, Fuller PJ, Gillespie MT, Milat F. Bone Disease in Thalassemia- A Molecular and Clinical Overview. Endocr Rev. 2016;37(4);320-346. doi: 10.1210/er.2015-1105 [Crossref][PubMed][Google Scholar]

12. Piga A. Impact of bone disease and pain in thalassemia. Hematology Am Soc Hematol Educ Program. 2017;2017(1)272-277. doi: 10.1182/asheducation-2017.1.272 [Crossref] [PubMed][Google Scholar]
13. Abbasi S, Asl JF, Zadeh LM, Mirdoraghi M. Measurement bone mineral density (BMD) of patients with beta thalassemia. Data Brief. 2018;19;1021-1024. doi: 10.1016/j.dib.2018.05.120 [Crossref][PubMed] [Google Scholar]

14. Shamshirsaz AA, Bekheirnia MR, Kamgar $M$, et al. Metabolic and endocrinologic complications in beta-thalassemia major- a multicenter study in Tehran. BMC Endocr Disord. 2003;3(1)4. doi: 10.1186/1472-6823-3-4 [Crossref][PubMed][Google Scholar]

15. Bazi A, Harati $H$, Khosravi-Bonjar A, Rakhshani E, Delaramnasab $M$. Hypothyroidism and Hypoparathyroidism in Thalassemia Major PatientsA Study in Sistan and Baluchestan Province, Iran. Int J Endocrinol Metab. 2018;16(2)e13228. doi: 10.5812\%2Fijem.13228 [Crossref][PubMed][Google Scholar]

16. Nawar FK, Gamal, et al. Bone Mineral Density and Calcium Status in Children with $\beta$-Thalassemia. J Am Sci. 2014;10(8)197-205. [Crossref][PubMed] [Google Scholar]

17. Dundar U, Kupesiz A, Ozdem S, Gilgil E, Tuncer $\mathrm{T}$, Yesilipek $\mathrm{A}$, et al. Bone metabolism and mineral density in patients with beta-thalassemia major. Saudi Med J. 2007;28(9):1425-1429. [Crossref] [PubMed][Google Scholar]

18. Aslan I, Canatan D, Balta N, Kacar G, Dorak C, Ozsancak $A$, et al. Bone mineral density in thalassemia major patients from Antalya, Turkey. Int J Endocrinol. 2012. doi: 10.1155/2012/573298 [Crossref][PubMed][Google Scholar] 